\title{
Revisiting an Underrecognized Strategy for Rhythm Management: Hybrid Therapy for Patients who Convert from Atrial Fibrillation to Flutter on Antiarrhythmic Drugs
}

\author{
FADY S. RIAD, MD ${ }^{1}$ and ALBERT L. WALDO, MD, PhD (Hon), FACC, FAHA, FHRS ${ }^{1}$ \\ ${ }^{1}$ Division of Cardiovascular Medicine, University Hospitals Cleveland Medical Center, Cleveland, OH, USA
}

\begin{abstract}
Atrial fibrillation (AF) is often treated with antiarrhythmic drugs (AADs) or catheter ablation. In a unique subset of patients, AF can convert to atrial flutter (AFL) after the initiation of an $A A D$. It has previously been shown that, in this subset of patients, cavotricuspid isthmus (CTI) ablation followed by the continuation of the AAD regimen has an unusually high rate of successfully maintaining sinus rhythm. This is an underrecognized approach toward rhythm management in such patients. However, the reason(s) for such a high degree of efficacy with this hybrid therapeutic approach are unclear. We suggest that conversion from AF to AFL selects for a group of patients in whom $A F$ is particularly responsive to the effects of the AAD. Since CTI ablation is essentially curative of AFL, the combination of both techniques results in a high efficacy of sinus rhythm maintenance. Further investigation is required to confirm these hypotheses.
\end{abstract}

KEYWORDS. Antiarrhythmic drugs, atrial fibrillation, atrial flutter, catheter ablation, class Ic antiarrhythmics.

\begin{abstract}
ISSN 2156-3977 (print) ISSN 2156-3993 (online)
\end{abstract} CC BY 4.0 license

C 2019 Innovations in Cardiac Rhythm Management

\section{Introduction}

Patients with atrial fibrillation $(\mathrm{AF})$ are most often treated with antiarrhythmic drugs (AADs) as a first-line strategy for rhythm control. As is well-documented, such treatment results in varying degrees of efficacy. Importantly, a subset of these patients will convert from AF to atrial flutter (AFL). It has been shown that, in patients treated with a class IC antiarrhythmic drug (AAD), as many as $20 \%$ will convert from AF to typical AFL. ${ }^{1}$ After successful cavotricuspid isthmus (CTI) ablation and, with continued

Dr. Waldo reports the reception of speaker's fees from Pfizer and Bristol-Myers Squibb, advisory board membership with Milestone Pharmaceuticals and Cardiac Insight, and clinical trial adverse outcomes adjudication committee membership with Biosense Webster, outside the scope of the submitted work. Dr. Riad reports no conflicts of interest for the published content.

Manuscript received October 16, 2018. Final version accepted February 11, 2019.

Address correspondence to: Albert L. Waldo, MD, PhD (Hon), FACC, FAHA, FHRS, 11100 Euclid Avenue, Cleveland, OH 44106, USA. Email: albert.waldo@case.edu. administration of their AAD, a remarkably high number of these patients will maintain sinus rhythm. Several small studies in the late 1990s to early 2000s evaluated patients with paroxysmal or chronic AF that converted to AFL after receiving an AAD-usually flecainide, another class IC agent, or amiodarone- and assessed arrhythmiafree survival after CTI ablation along with maintaining therapy with the AAD. Their results showed remarkable efficacy for the long-term maintenance of sinus rhythm, including rates of up to $80 \%$ to $90 \%$ in some. Others showed more modest results for arrhythmia-free survival, but all highlighted a substantial decrease in symptomatic recurrence and frequency of recurrence (Table 1). ${ }^{2-6}$ This strategy, however, is underrecognized in current clinical practice, and no large prospective trials of this method of treatment exist at this time. Nevertheless, we suggest that consideration of this approach for treating AF, particularly in patients with new-onset or paroxysmal $\mathrm{AF}$, should be given as long as there are no contraindications to using a class Ic antiarrhythmic in a particular case. Finally, the reasons for the unique effectiveness of 


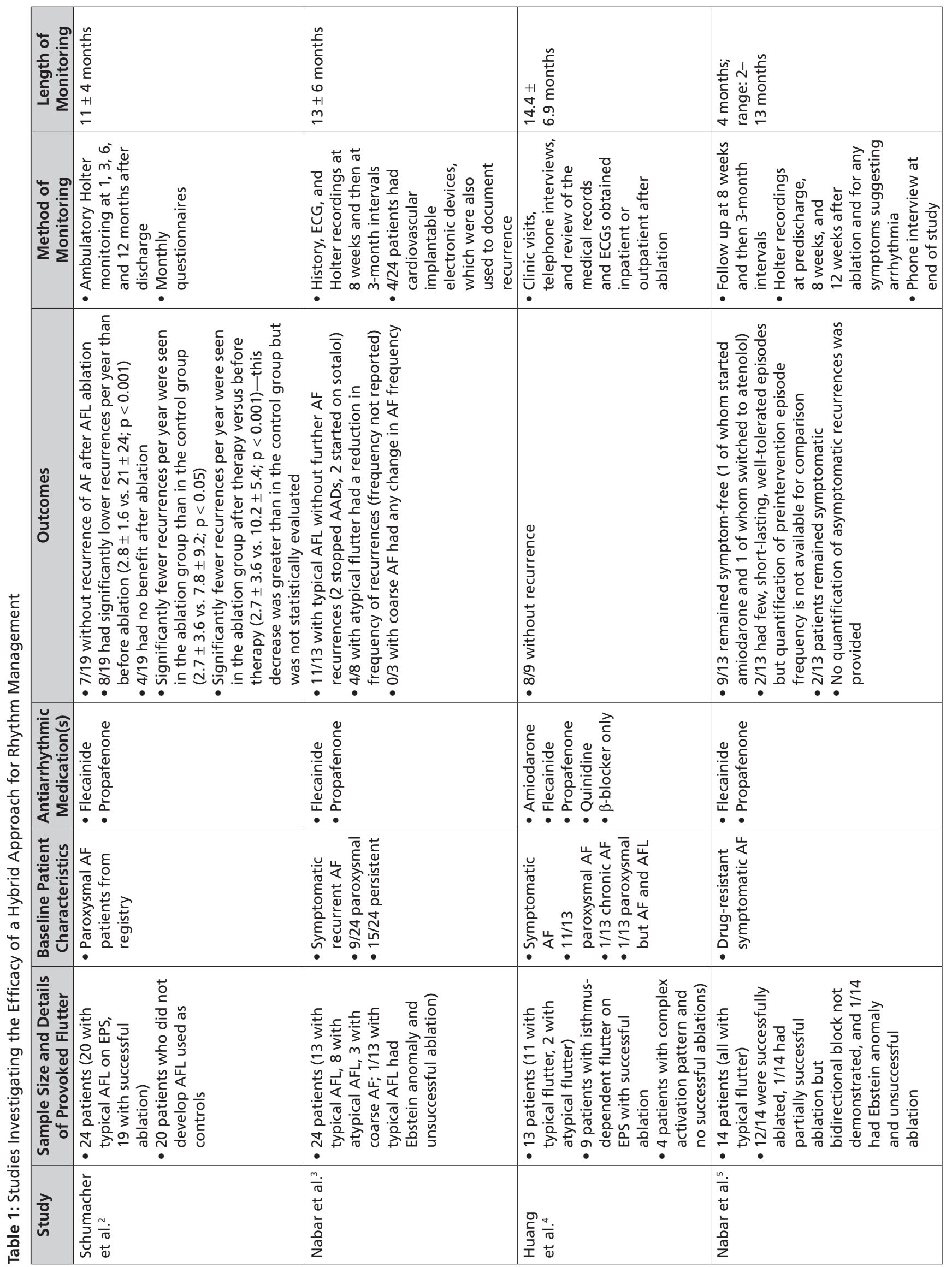




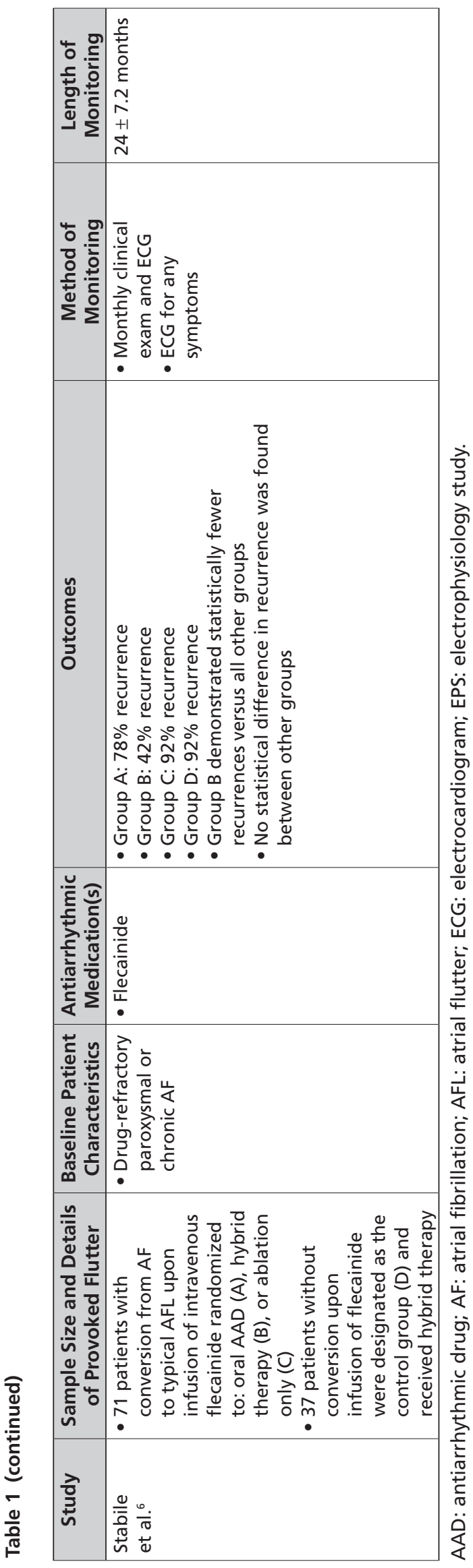

this hybrid approach to treating AF are unknown. What follows thus is our attempt to understand the whys and wherefores of this unique mode of therapy.

\section{Role of class Ic antiarrhythmics for the restoration of sinus rhythm in the general population}

Flecainide is an effective medication for the cardioversion of $\mathrm{AF}$, resulting in a $70 \%$ to $90 \%$ rate of conversion to sinus rhythm within eight hours of administration. ${ }^{7-13}$ It has also been shown to significantly reduce the recurrence of $\mathrm{AF}$ as well as prolong the time to first $\mathrm{AF}$ recurrence and the time interval between AF recurrences. ${ }^{14-17}$ The rate of AF recurrence within several months to one year has been shown to be between $30 \%$ and $70 \%$ among various studies, although, in patients deemed "difficult to treat," the effect was predominantly an increase in the time between AF recurrences, with no significant impact on total burden of AF recurrence. ${ }^{15}$ Propafenone, another class Ic agent, has been shown to have similar efficacy. ${ }^{12,13,18,19}$ This class of agents, however, has been associated with seemingly "proarrhythmic" effectsnamely, conversion to a relatively slow AFL-that, when slow enough, may result in 1:1 atrioventricular (AV) conduction in the absence of sufficient AV nodal blockade. The exact incidence of this phenomenon is unclear, as most reports are in the form of case reports or small case series. ${ }^{20-22}$ Fortunately, this symptomatic conversion is rare and the adequate use of $\mathrm{AV}$ node-blocking agents should prevent its occurrence. . $^{1,23}$

\section{A hybrid approach to rhythm management in patients with atrial fibrillation that converts to typical atrial flutter on class Ic agents}

Some have suggested this "proarrhythmic" effect of AADs (ie, conversion of AF to AFL) to be less of an obstacle and more so an opportunity, hypothesizing that, when such patients converted from AF to typical AFL, the addition of CTI ablation would not only address the treatment of AFL but also enhance the suppression of AF. As noted above, this clinical approach demonstrated a very high rate of efficacy when AF converted to typical AFL. ${ }^{2-6}$

Understanding these clinical outcomes, however, requires comprehension of the relationship between AF and typical AFL. This relationship has long been documented in the literature. ${ }^{24,25}$ Specifically, it has been noted that AFL rarely forms without a preceding episode of $\mathrm{AF}^{26}$ Such $\mathrm{AF}$ episodes are often underrecognized because they are typically short, lasting only seconds to minutes. ${ }^{27}$ These observations are bolstered by the finding that, in patients with concurrent AF and typical AFL, successful pulmonary vein isolation (PVI) alone was as effective as PVI plus CTI ablation for the prevention of AFL recurrence. ${ }^{28}$ This finding supports the notion that $\mathrm{AF}$ is the trigger for AFL, since elimination of the AF by PVI alone resulted in elimination of the AFL as well. 


\section{Physiology of the development of atrial flutter}

Another important aspect of the development of typical AFL is the formation of a functional line of block in the right atrial free wall between the venae cavae, which is necessary to prevent short-circuiting of the macroreentrant AFL circuit. This was initially demonstrated in a canine model, which showed that, in all episodes of AFL, such a line of functional block was developed. ${ }^{29}$ Furthermore, it was demonstrated that, when this line of block decreased in length to a critical value, the AFL converted to AF, consistent with the notion that this line of block was critical for the maintenance of typical AFL (Figure 1). These findings have been confirmed through invasive electrophysiologic studies of $\mathrm{AF}$ in humans and the line of block was localized to the crista terminalis. ${ }^{30-32}$ Notably, it was shown that this line of block developed in a rate-dependent manner, with shorter AF cycle lengths being more likely to result in conduction block. ${ }^{32,33}$ Importantly, the cycle length required to maintain the line of block in patients with AF was significantly shorter than that in patients with AFL. ${ }^{33,34}$ This finding is critical because if the rate required to maintain the line of block was significantly faster than the rate of the resulting AFL circuit, the line of block could not be maintained and the reentrant circuit would degenerate into $\mathrm{AF}$ once more. This concept of the importance of intercaval block is supported by findings that patients after the surgical repair of an atrial septal defect often present with AFL, not $\mathrm{AF}^{35,36}$ We suggest that this occurs because the fixed line of intercaval block from the surgical scar is present to maintain the reentrant circuit. Finally, it has been shown that antiarrhythmic therapy with amiodarone can also promote the development of this line of block, associated with the development of AFL. ${ }^{34}$

Synthesis of the above findings suggests that the development of typical AFL begins with an episode of AF that, due to rapid atrial rates, leads to the onset of a functional line of block along the crista terminalis (essentially a block between the superior and inferior venae cavae). In the right patient, the rate required for formation of this functional block is slow enough that, once the AFL circuit forms, it is able to sustain the line of block on its own. Antiarrhythmics, especially those that slow conduction through the atrial myocardium or increase the atrial refractory period, may decrease the threshold for the formation of this line of block and, therefore, promote the conversion of AF to AFL. There is additionally some evidence that short delays in electrical activity in the right atrial free wall are ubiquitously seen immediately preceding the conversion of AF to AFL. ${ }^{27}$ This period potentially allows for or is a result of the organization of the various activation wavefronts in AF and enables the formation of a single wavefront that becomes the AFL reentrant circuit. Although this finding requires further confirmation, it could be the final piece of evidence needed to explain why some patients convert to AFL and others do not. Essentially, once the requisite conditions for the formation of AFL are obtained, there is a component of chance that the multiple wavefronts during AF will interact in such a way that they form a functional intercaval line of block as well as a unified wavefront that will circulate
A

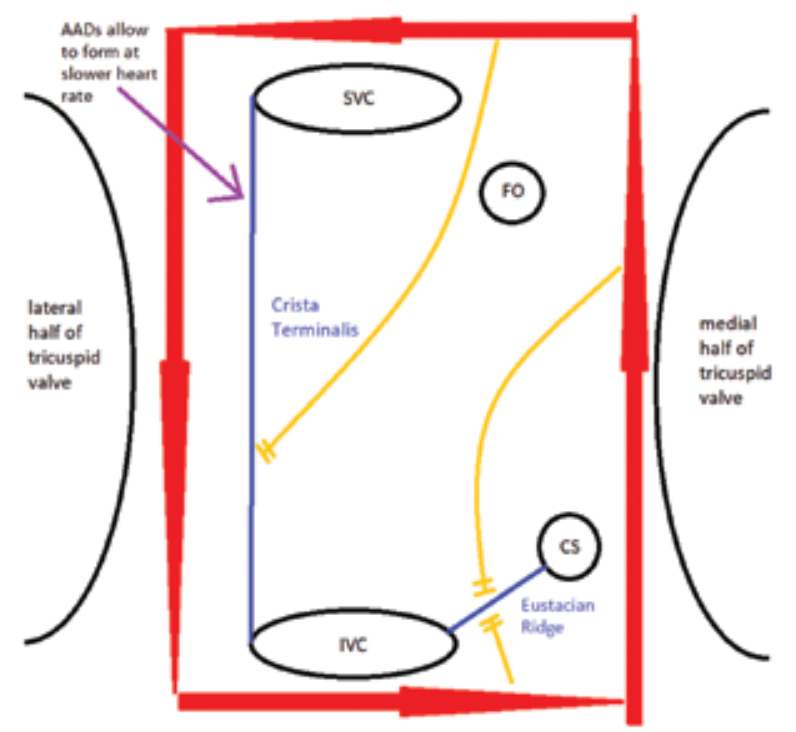

B

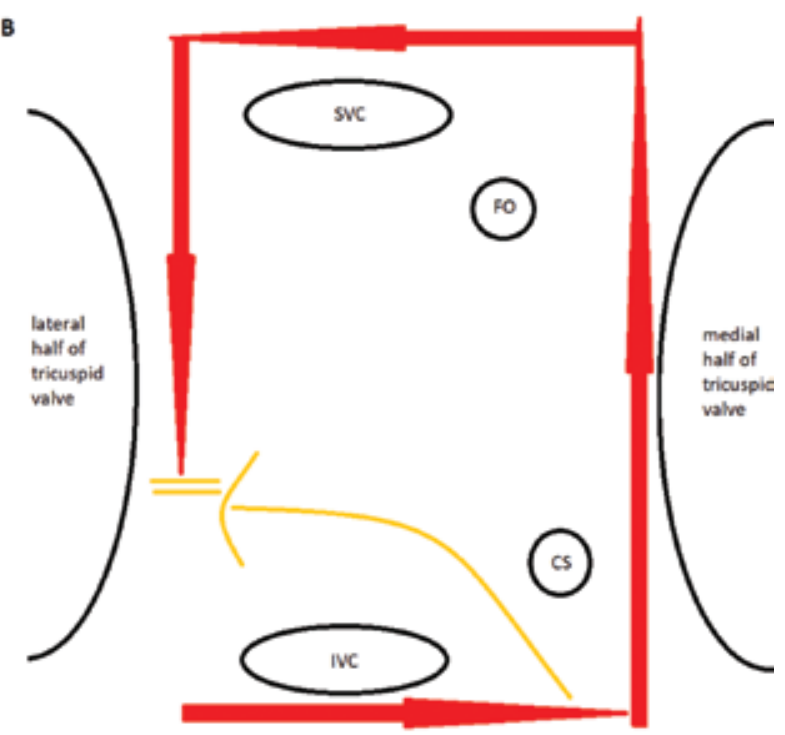

Figure 1: View of the right atrium from a bisected tricuspid valve toward the posterior wall. A: AFL circuit (red arrows) traversing in a counterclockwise direction around the tricuspid valve. Functional lines of block primarily along the crista terminalis and also the eustachian ridge (blue lines) prevent short-circuiting of the circuit (yellow lines). AADs promote the formation of these lines by reducing the threshold rate for formation to below the rate of the AFL circuit. B: AFL circuit (red arrows) traversing in a counterclockwise direction around the tricuspid valve. The lack of a line of block between the venae cavae allows for short-circuiting of the circuit around the superior vena cava or inferior vena cava (yellow arrow), causing termination of the macroreentrant circuit. 
around it and form the AFL reentrant circuit. Statistically, this would occur sooner or later and would primarily be a function of time.

\section{Role of class Ic agents in the hybrid strategy for rhythm management}

Notably, while an understanding of the above can explain why we see conversion from AF to AFL in some patients receiving class IC antiarrhythmic agents, this does not explain why there is such a high rate of success in maintaining sinus rhythm after CTI ablation with continued administration of an AAD. Several explanations for this phenomenon can be considered. We suggest that the most compelling mechanism is that conversion from AF to typical AFL upon the initiation of class Ic antiarrhythmics selects for a subset of patients who are more likely to respond to the medication itself. Alternatively, this may be an incidental finding. Class Ic antiarrhythmics are already effective agents in maintaining sinus rhythm, as described above. It has also been shown that CTI ablation alone can sometimes reduce the incidence of further $\mathrm{AF}^{37}$ The combined use of both modalities may simply have an additive effect on the maintenance of sinus rhythm. We find this to be a less-compelling explanation, however, given the magnitude of the difference in efficacy between the hybrid approach and the effect of the sum of individual approaches. ${ }^{6}$ Given the small size of studies reporting on use of this combined approach for rhythm management in AF, further investigation is still needed to fully describe the reasons for the high efficacy of this technique. Should findings from such research support our primary hypothesis that conversion from AF to AFL using class Ic agents selects for patients more likely to respond to the medication, it would be interesting to then determine whether or not different subsets of patients are more likely to respond to different antiarrhythmics. Ultimately, such an investigation could lead to the discovery of clinical or anatomic factors that could help to guide the choice of antiarrhythmic agent. We suggest that studies should be focused on the ability of various medications to promote the formation of a functional line of block along the crista terminalis.

\section{Conclusion}

AF and AFL are highly related arrhythmias. It has long been noted that class Ic antiarrhythmic agents can promote the conversion of AF to AFL. This phenomenon occurs because rapid atrial rates during AF result in the formation of a functional line of block along the crista terminalis, creating a substrate for the macroreentrant circuit of AFL. AADs may decrease the threshold for the formation of this line of block, enabling it to be stable at slower atrial rates and, therefore, capable of being maintained by the AFL circuit itself. A hybrid approach to the treatment of AF has been suggested that involves CTI ablation and the maintenance of the antiarrhythmic medication for patients who initially convert from AF to typical AFL. This strategy has been shown to have a high success rate in limited studies. The reason for this high efficacy is unknown. We suggest that conversion from AF to AFL with class IC antiarrhythmics selects for a subset of the population in whom these medications are highly effective. Understanding this phenomenon may lead to better medication selection for patients or the development of more effective antiarrhythmic medications in the future. We also recommend that this scenario should not necessarily be viewed as a failure of the AAD. Despite advances in ablation technology, PVI remains a more invasive procedure with higher risks than isolated CTI ablation. While there is not yet enough evidence to recommend routinely omitting PVI in these patients, we feel that, in select patients, a trial of CTI ablation without PVI plus continuation of the current AAD is warranted and may result in fewer complications.

\section{References}

1. Bianconi L, Mennuni M, Lukic V, Castro A, Chieffi M, Santini M. Effects of oral propafenone administration before electrical cardioversion of chronic atrial fibrillation: a placebo-controlled study. J Am Coll Cardiol. 1996;28(3):700-706.

2. Schumacher B, Jung W, Lewalter T, Vahlhaus C, Wolpert C, Lüderitz B. Radiofrequency ablation of atrial flutter due to administration of class IC antiarrhythmic drugs for atrial fibrillation. Am J Cardiol. 1999;83(5):710-703.

3. Nabar A, Rodriguez LM, Timmermans C, van Mechelen $\mathrm{R}$, Wellens HJ. Class IC antiarrhythmic drug induced atrial flutter: electrocardiographic and electrophysiological findings and their importance for long term outcome after right atrial isthmus ablation. Heart. 2001;85(4):424-429.

4. Huang DT, Monahan KM, Zimetbaum P, Papageorgiou P, Epstein LM, Josephson ME. Hybrid pharmacologic and ablative therapy: a novel and effective approach for the management of atrial fibrillation. J Cardiovasc Electrophysiol. 1998;9(5):462-469.

5. Nabar A, Rodriguez LM, Timmermans C, Smeets JL, Wellens HJ. Radiofrequency ablation of "class IC atrial flutter" in patients with resistant atrial fibrillation. Am J Cardiol. 1999;83(5):785-787, A10.

6. Stabile G, De Simone A, Turco P, et al. Response to flecainide infusion predicts long-term success of hybrid pharmacologic and ablation therapy in patients with atrial fibrillation. J Am Coll Cardiol. 2001;37(6):1639-1644.

7. Capucci A, Lenzi T, Boriani G, et al. Effectiveness of loading oral flecainide for converting recent-onset atrial fibrillation to sinus rhythm in patients without organic heart disease or with only systemic hypertension. Am J Cardiol. 1992;70(1):69-72.

8. Donovan KD, Dobb GJ, Coombs LJ, et al. Efficacy of flecainide for the reversion of acute onset atrial fibrillation. Am J Cardiol. 1992;70(5):50A-54A; discussion 54A-55A.

9. Donovan KD, Power BM, Hockings BE, Dobb GJ, Lee KY. Intravenous flecainide versus amiodarone for recent-onset atrial fibrillation. Am J Cardiol. 1995;75(10):693-697.

10. Boriani G, Biffi M, Capucci A, et al. Conversion of recent-onset atrial fibrillation to sinus rhythm: effects of different drug protocols. Pacing Clin Electrophysiol. 1998;21(11 Pt 2):2470-2474.

11. Martínez-Marcos FJ, García-Garmendia JL, Ortega-Carpio A, Fernández-Gómez JM, Santos JM, Camacho C. Comparison of intravenous flecainide, propafenone, and amiodarone for conversion of acute atrial fibrillation to sinus rhythm. Am J Cardiol. 2000;86(9):950-953. 
12. Romano S, Fattore L, Toscano G, et al. [Effectiveness and side effects of the treatment with propafenone and flecainide for recent-onset atrial fibrillation]. Ital Heart J Suppl. 2001;2(1):41-45. Article in Italian.

13. Alboni P, Botto GL, Baldi N, et al. Outpatient treatment of recent-onset atrial fibrillation with the "pill-in-the-pocket" approach. N Engl J Med. 2004;351(23):2384-2391.

14. Anderson JL, Gilbert EM, Alpert BL, et al. Prevention of symptomatic recurrences of paroxysmal atrial fibrillation in patients initially tolerating antiarrhythmic therapy. A multicenter, double-blind, crossover study of flecainide and placebo with transtelephonic monitoring. Flecainide Supraventricular Tachycardia Study Group. Circulation. 1989;80(6):1557-1570.

15. Van Gelder IC, Crijns HJ, Van Gilst WH, Van Wijk LM, Hamer HP, Lie KI. Efficacy and safety of flecainide acetate in the maintenance of sinus rhythm after electrical cardioversion of chronic atrial fibrillation or atrial flutter. Am J Cardiol. 1989;64(19):1317-1321.

16. Pietersen $\mathrm{AH}, \mathrm{Hellemann} \mathrm{H}$. Usefulness of flecainide for prevention of paroxysmal atrial fibrillation and flutter. Danish-Norwegian Flecainide Multicenter Study Group. Am J Cardiol. 1991;67(8):713-717.

17. Carunchio A, Fera MS, Mazza A, et al. [A comparison between flecainide and sotalol in the prevention of recurrences of paroxysmal atrial fibrillation]. G Ital Cardiol. 1995;25(1):51-68. Article in Italian.

18. Chimienti M, Cullen MT, Casadei G. Safety of flecainide versus propafenone for the long-term management of symptomatic paroxysmal supraventricular tachyarrhythmias. Report from the Flecainide and Propafenone Italian Study (FAPIS) Group. Eur Heart J. 1995;16(12):1943-1951.

19. Lafuente-Lafuente C, Mouly S, Longás-Tejero MA, Mahé I, Bergmann J. Antiarrhythmic drugs for maintaining sinus rhythm after cardioversion of atrial fibrillation: a systematic review of randomized controlled trials. Arch Intern Med. 2006;166(7):719-728.

20. Comelli I, Pigna F, Cervellin G. 1:1 atrial flutter induced by flecainide, whilst the patient was at rest. Am J Emerg Med. 2018;36(11):2131.e3-2131.e5.

21. Taylor R, Gandhi MM, Lloyd G. Tachycardia due to atrial flutter with rapid 1:1 conduction following treatment of atrial fibrillation with flecainide. BMJ. 2010;340:b4684.

22. Feld GK, Chen PS, Nicod P, Fleck RP, Meyer D. Possible atrial proarrhythmic effects of class 1C antiarrhythmic drugs. Am J Cardiol. 1990;66(3):378-383.

23. Falk RH. Proarrhythmic responses to atrial antiarrhythmic therapy. In: Falk RH, Podrid PJ, eds. Atrial Fibrillation: Mechanisms and Management. New York, NY: Raven Press; 1992: 283-305.

24. Waldo AL. Inter-relationships between atrial flutter and atrial fibrillation. Pacing Clin Electrophysiol. 2003;26:1583-1596.
25. Waldo AL, Feld GK. Interrelationships of atrial fibrillation and atrial flutter. Mechanisms and clinical implications. $J$ Am Coll Cardiol. 2008;51(8):779-786.

26. Waldo AL. Atrial fibrillation and atrial flutter: Two sides of the same coin!. Int J Cardiol. 2017;240:251-252.

27. Roithinger FX, Karch MR, Steiner PR, SippensGroenewegen A, Lesh MD. Relationship between atrial fibrillation and typical atrial flutter in humans: activation sequence changes during spontaneous conversion. Circulation. 1997;96(10):3484-3491.

28. Wazni O, Marrouche NF, Martin DO, et al. Randomized study comparing combined pulmonary vein-left atrial junction disconnection and cavotricuspid isthmus ablation versus pulmonary vein-left atrial junction disconnection alone in patients presenting with typical atrial flutter and atrial fibrillation. Circulation. 2003;108(20):2479-2483.

29. Ortiz J, Niwano S, Abe H, Rudy Y, Johnson NJ, Waldo AL. Mapping the conversion of atrial flutter to atrial fibrillation and atrial fibrillation to atrial flutter. Insights into mechanisms. Circ Res. 1994;74:882-894.

30. Olgin JE, Kalman JM, Fitzpatrick AP, Lesh MD. Role of right atrial endocardial structures as barriers to conduction during human type I atrial flutter. Activation and entrainment mapping guided by intracardiac echocardiography. Circulation. 1995;92(7):1839-1848.

31. Olgin JE, Kalman JM, Lesh MD. Conduction barriers in human atrial flutter: correlation of electrophysiology and anatomy. J Cardiovasc Electrophysiol. 1996;7(11):1112-1126.

32. Yamabe H, Misumi I, Fukushima H, Ueno K, Kimura Y, Hokamura Y. Conduction properties of the crista terminalis and its influence on the right atrial activation sequence in patients with typical atrial flutter. Pacing Clin Electrophysiol. 2002;25(2):132-141.

33. Schumacher B, Jung W, Schmidt H, et al. Transverse conduction capabilities of the crista terminalis in patients with atrial flutter and atrial fibrillation. J Am Coll Cardiol. 1999;34(2):363-373.

34. Tai CT, Lin YK, Lan FC, et al. Conduction properties of the crista terminalis in patients with atrial flutter due to amiodarone therapy for atrial fibrillation. Pacing Clin Electrophysiol. 2003;26(12):2241-2246.

35. Combes N, Derval N, Hascoët S, et al. Ablation of supraventricular arrhythmias in adult congenital heart disease: a contemporary review. Arch Cardiovasc Dis. 2017;110(5):334-345.

36. Chan DP, Van Hare GF, Mackall JA, Carlson MD, Waldo AL. Importance of atrial flutter isthmus in postoperative intra-atrial reentrant tachycardia. Circulation. 2000;102(11):1283-1289.

37. Schmieder S, Ndrepepa G, Dong J, et al. Acute and longterm results of radiofrequency ablation of common atrial flutter and the influence of the right atrial isthmus ablation on the occurrence of atrial fibrillation. Eur Heart J. 2003;24(10):956-962. 